\title{
Boundary following using gyroscopic control
}

\author{
F. Zhang, E. W. Justh \\ Institute for Systems Research \\ University of Maryland \\ College Park, MD 20742, USA \\ fuminz@isr.umd.edu, \\ justheisr.umd.edu
}

\author{
P. S. Krishnaprasad \\ Institute for Systems Research and \\ Dept. of Electrical and Computer Engineering \\ University of Maryland \\ College Park, MD 20742, USA \\ krishnaeisr.umd.edu
}

\begin{abstract}
Recent work in the study of interacting particles has demonstrated the effectiveness of gyroscopic interactions in producing desired stable spatial patterns (formations) of motion of a collective of particles. In this paper, we discuss the problem of how a single particle might interact with a fixed structure in space by exploiting gyroscopic feedback laws. We derive a gyroscopic feedback law modeling the interaction of a particle in the plane with an image particle representing the closest point on a simple closed curve bounding an obstacle and show that this law produces boundary-following behavior. We also provide a preliminary discussion of the three-dimensional case.
\end{abstract}

\section{INTRODUCTION}

We consider a vehicle moving at unit speed in the presence of an obstacle, and the prototype problem we consider is boundary-following with collision avoidance. In the plane, the moving vehicle is subject to steering (i.e., curvature) control. From the point of view of mechanics, we are considering particle motion subject to gyroscopic forces; i.e., forces which change the direction of motion of the particle without altering its kinetic energy (and hence its speed). Recently, the idea of using gyroscopic forces for obstacle avoidance in robotics has gained renewed attention [1], [2], [3], and similar ideas have also started appearing in behavioral psychology [4]. In this work, we draw strong analogies with recent work on two-vehicle interaction laws developed for formation control [5], [6] to develop a novel formulation of a gyroscopic boundaryfollowing and collision avoidance law. In the planar setting, we prove global convergence results for circular and linear obstacle boundaries. The key calculations are also shown to carry over to the three-dimensional setting, but a rigorous proof of convergence in three dimensions is left for a future paper.

\section{BOUNDARY-FOLLOWING MODEL}

\section{A. Dynamical equations}

In the planar setting, consider a vehicle moving at unit speed (and subject to steering control) in the presence of a single obstacle (i.e., the region enclosed by a simple closed curve) whose boundary is twice continuously differentiable. Suppose that at each instant of time, the point on the obstacle boundary which is closest to (i.e., the minimum Euclidean distance from) the moving vehicle is unique. This point on the obstacle boundary, which we will call the "closest point" (or "shadow point"), moves along the boundary curve. (We assume uniqueness of the closest point in order to streamline the discussion and bring out the key ideas. Of course, in dealing with real-world obstacles, nonuniqueness of the closest point is an important issue.)

Let $\mathbf{r}_{1}$ denote the position of the closest point, let $\mathbf{x}_{1}$ denote the unit tangent vector to the boundary curve at the closest point, and let $\mathbf{y}_{1}$ denote the unit normal vector. We use the convention that a unit normal vector completes a right-handed orthonormal frame with the corresponding unit tangent vector. In terms of the arc-length parameterization, the boundary curve can be described by

$$
\begin{aligned}
\mathbf{r}_{1}^{\prime} & =\mathbf{x}_{1}, \\
\mathbf{x}_{1}^{\prime} & =\mathbf{y}_{1} \kappa_{1}, \\
\mathbf{y}_{1}^{\prime} & =-\mathbf{x}_{1} \kappa_{1},
\end{aligned}
$$

where the prime denotes differentiation with respect to arclength parameter, and $\kappa_{1}$ is the plane curvature function for the boundary curve. Using the chain rule, we can express the time-evolution of the closest point as

$$
\begin{aligned}
& \dot{\mathbf{r}}_{1}=\nu_{1} \mathbf{x}_{1}, \\
& \dot{\mathbf{x}}_{1}=\mathbf{y}_{1} \nu_{1} \kappa_{1}, \\
& \dot{\mathbf{y}}_{1}=-\mathbf{x}_{1} \nu_{1} \kappa_{1},
\end{aligned}
$$

where

$$
\nu_{1}=\frac{d s}{d t}
$$

with $s$ denoting the arc-length parameter. Because the closest point depends on the motion of the moving vehicle, $\nu_{1}$ depends on both the boundary curve and on the trajectory of the moving vehicle.

Letting $\mathbf{r}_{2}$ denote the position of the moving vehicle, $\mathbf{x}_{2}$ the unit tangent vector, $\mathbf{y}_{2}$ the unit normal vector, and $u_{2}$ the steering control for the moving vehicle, we have the following system of equations for the "formation" consisting of the moving vehicle and the closest point:

$$
\begin{array}{ll}
\dot{\mathbf{r}}_{1}=\nu_{1} \mathbf{x}_{1}, & \dot{\mathbf{r}}_{2}=\mathbf{x}_{2}, \\
\dot{\mathbf{x}}_{1}=\mathbf{y}_{1} \nu_{1} \kappa_{1}, & \dot{\mathbf{x}}_{2}=\mathbf{y}_{2} u_{2}, \\
\dot{\mathbf{y}}_{1}=-\mathbf{x}_{1} \nu_{1} \kappa_{1}, & \dot{\mathbf{y}}_{2}=-\mathbf{x}_{2} u_{2},
\end{array}
$$

where $\kappa_{1}$ may be considered given (in practice, $\kappa_{1}$ is derived from sensor data, e.g., from a laser rangefinder); $\nu_{1}$ is a deterministic function of $\left(\mathbf{r}_{1}, \mathbf{x}_{1}, \mathbf{y}_{1}\right),\left(\mathbf{r}_{2}, \mathbf{x}_{2}, \mathbf{y}_{2}\right)$, and $\kappa_{1}$; and $u_{2}$ is the control input we apply to avoid colliding with 


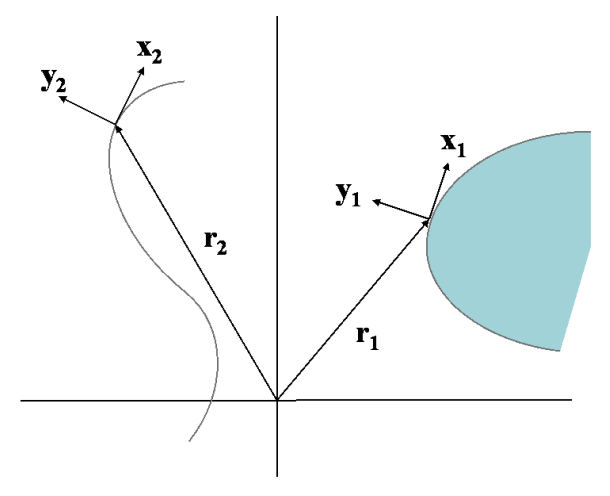

Fig. 1. Positions and frames for the trajectory of the moving vehicle $\left(\mathbf{r}_{2}\right.$, $\left.\mathbf{x}_{2}, \mathbf{y}_{2}\right)$ and for the closest point on the boundary curve $\left(\mathbf{r}_{1}, \mathbf{x}_{1}, \mathbf{y}_{1}\right)$.

the obstacle and to achieve boundary following (see figure 1). Our objective is to determine $u_{2}$, as a feedback function of $\left(\mathbf{r}_{1}, \mathbf{x}_{1}, \mathbf{y}_{1}\right),\left(\mathbf{r}_{2}, \mathbf{x}_{2}, \mathbf{y}_{2}\right)$, and $\kappa_{1}$, such that we can prove analytically that collision avoidance and boundary following are achieved. Here we focus on certain special cases - linear boundary curves and circular boundary curves - because the analysis is more straightforward for these special cases than for general boundary curves, while the key ideas are still adequately illustrated.

\section{B. Boundary-curve frame convention}

We define

$$
\mathbf{r}=\mathbf{r}_{2}-\mathbf{r}_{1}
$$

to be the vector from the closest point on the boundary curve to the moving vehicle. We assume that initially

$$
|\mathbf{r}|>0
$$

and we will prove that under this assumption (as well as other appropriate hypotheses), our boundary-following control law guarantees (6) for all future time. Then

$$
\begin{aligned}
\frac{d}{d t}|\mathbf{r}| & =\frac{\mathbf{r} \cdot \dot{\mathbf{r}}}{|\mathbf{r}|}=\frac{\mathbf{r}}{|\mathbf{r}|} \cdot\left(\mathbf{x}_{2}-\nu_{1} \mathbf{x}_{1}\right) \\
& =\left(\frac{\mathbf{r}}{|\mathbf{r}|} \cdot \mathbf{x}_{2}\right)-\nu_{1}\left(\frac{\mathbf{r}}{|\mathbf{r}|} \cdot \mathbf{x}_{1}\right) .
\end{aligned}
$$

The first-order necessary conditions for the closest point to be an extremum of the Euclidean distance from the moving vehicle to the curve are

$$
\mathbf{r} \cdot \mathbf{x}_{1} \equiv 0
$$

and

$$
\mathbf{y}_{1} \equiv \pm \frac{\mathbf{r}}{|\mathbf{r}|},
$$

where the correct choice of sign depends on whether the boundary curve is to the right or left of the moving vehicle, and on what convention is chosen for the positive direction along the boundary curve.

In order to fix a convention for the positive direction of the boundary curve, we assume that initially

$$
\mathbf{r} \cdot \mathbf{y}_{2} \neq 0
$$

i.e., that the moving vehicle is not initially heading directly toward or directly away from the closest point on the boundary curve. We will prove that under this assumption (and other appropriate hypotheses), our boundary-following control law guarantees (10) for all future time. We may thus choose the positive direction of the boundary curve such that

$$
\mathbf{x}_{1} \cdot \mathbf{x}_{2}>0 \text {. }
$$

With this convention, the choice of sign in (9) is determined by whether the boundary curve is to the left or right of the moving vehicle. Furthermore, because of our convention that $\left(\mathbf{x}_{1}, \mathbf{y}_{1}\right)$ forms a right-handed frame, (11) implies that the sign of $\kappa_{1}$ depends not just on the boundary curve, but on the position and orientation of the moving vehicle, as well. (Although curvature is a property of an oriented boundary curve alone, we are allowing the orientation of the boundary curve to depend on the relative position and orientation of the moving vehicle.)

Using (7) with (8) and (9), we obtain

$$
\frac{d}{d t}|\mathbf{r}|=\left(\frac{\mathbf{r}}{|\mathbf{r}|} \cdot \mathbf{x}_{2}\right)= \pm \mathbf{y}_{1} \cdot \mathbf{x}_{2}=\mp \mathbf{x}_{1} \cdot \mathbf{y}_{2}
$$

We can derive an expression for $\nu_{1}$ by differentiating (8) with respect to time, to obtain

$$
\begin{aligned}
\frac{d}{d t}\left(\mathbf{r} \cdot \mathbf{x}_{1}\right) & =\dot{\mathbf{r}} \cdot \mathbf{x}_{1}+\mathbf{r} \cdot \dot{\mathbf{x}}_{1} \\
& =\left(\mathbf{x}_{2}-\nu_{1} \mathbf{x}_{1}\right) \cdot \mathbf{x}_{1}+\left(\frac{\mathbf{r}}{|\mathbf{r}|} \cdot \mathbf{y}_{1}\right)|\mathbf{r}| \nu_{1} \kappa_{1} \\
& =\mathbf{x}_{1} \cdot \mathbf{x}_{2}-\nu_{1} \pm|\mathbf{r}| \nu_{1} \kappa_{1} \\
& =0
\end{aligned}
$$

where we have used equations (8) and (9). We then have

$$
\left(1 \mp|\mathbf{r}| \kappa_{1}\right) \nu_{1}=\mathbf{x}_{1} \cdot \mathbf{x}_{2},
$$

and for simplicity we replace $\mp \kappa_{1}$ with $\pm\left|\kappa_{1}\right|$, leading, finally, to

$$
\nu_{1}=\frac{\mathbf{x}_{1} \cdot \mathbf{x}_{2}}{1 \pm\left|\kappa_{1}\right||\mathbf{r}|}>0
$$

where we assume $1 \pm\left|\kappa_{1}\right||\mathbf{r}|>0$. If $\left(\frac{\mathbf{r}}{|\mathbf{r}|} \cdot \mathbf{y}_{1}\right) \kappa_{1}<0$, we say that the boundary "curves away from" the moving vehicle, and if $\left(\frac{\mathbf{r}}{|\mathbf{r}|} \cdot \mathbf{y}_{1}\right) \kappa_{1}>0$, we say that the boundary "curves toward" the moving vehicle. (The intermediate case corresponds to $\kappa_{1}=0$.) In (15), the plus sign is used when the boundary curves away from the moving vehicle, and the minus sign is used when the boundary curves inward toward the moving vehicle. In the latter case, we note that there is a singularity in the expression for $\nu_{1}$ when $|\mathbf{r}|=1 /\left|\kappa_{1}\right|$ and the boundary curves inward toward the moving vehicle.

\section{Shape variables}

We would like to treat the moving vehicle and the closest point on the boundary as two interacting "vehicles" - one moving at unit speed, and the other not necessarily moving at unit speed - and then demonstrate analytically 
that the motion of the two "vehicles" converges to a steadystate "formation." This approach is easiest to describe if appropriate "shape variables," which depend only on the relative positions and orientations of the "vehicles," can be identified. The formation is then an equilibrium for the shape dynamics, and is therefore a relative equilibrium for the (possibly nonautonomous) dynamics (4).

Here we identify the shape variables $|\mathbf{r}|$ and $\mathbf{x}_{1} \cdot \mathbf{y}_{2}$. Note that we can define the angle $\phi$ between the heading direction of the moving vehicle and the tangent vector to the boundary curve at the closest point according to

$$
\mathbf{x}_{1} \cdot \mathbf{y}_{2}=\sin \phi
$$

let $\rho=|\mathbf{r}|$, and instead use $(\rho, \phi)$ as shape variables. Note that because

$$
\mathbf{x}_{1} \cdot \mathbf{x}_{2}=\cos \phi
$$

and (11) holds, in fact

$$
-\frac{\pi}{2}<\phi<\frac{\pi}{2}
$$

We can express the shape dynamics as follows. From (12) we have

$$
\dot{\rho}=\mp \sin \phi .
$$

Differentiating $\mathbf{x}_{1} \cdot \mathbf{y}_{2}$ with respect to time gives

$$
\begin{aligned}
\frac{d}{d t}\left(\mathbf{x}_{1} \cdot \mathbf{y}_{2}\right) & =\dot{\mathbf{x}}_{1} \cdot \mathbf{y}_{2}+\mathbf{x}_{1} \cdot \dot{\mathbf{y}}_{2} \\
& =\nu_{1} \kappa_{1}\left(\mathbf{y}_{1} \cdot \mathbf{y}_{2}\right)-u_{2}\left(\mathbf{x}_{1} \cdot \mathbf{x}_{2}\right) \\
& =\left[\left(\frac{\mathbf{x}_{1} \cdot \mathbf{x}_{2}}{1 \pm\left|\kappa_{1}\right||\mathbf{r}|}\right) \kappa_{1}-u_{2}\right]\left(\mathbf{x}_{1} \cdot \mathbf{x}_{2}\right) \\
& =\left[\left(\frac{\kappa_{1}}{1 \pm\left|\kappa_{1}\right| \rho}\right) \cos \phi-u_{2}\right] \cos \phi,
\end{aligned}
$$

where we have used (15). Since we also have

$$
\frac{d}{d t}\left(\mathbf{x}_{1} \cdot \mathbf{y}_{2}\right)=\frac{d}{d t}(\sin \phi)=(\cos \phi) \dot{\phi}
$$

we obtain

$$
\dot{\phi}=\left(\frac{\kappa_{1}}{1 \pm\left|\kappa_{1}\right| \rho}\right) \cos \phi-u_{2} .
$$

Thus, provided $u_{2}=u_{2}(\rho, \phi)$, we see that the shape dynamics consisting of (19) together with (22) are selfcontained. If $\kappa_{1}=$ constant, then the shape dynamics are also autonomous. However, even if $\kappa_{1}$ is nonconstant, below we show that, under certain hypotheses, the control $u_{2}$ may be chosen so as to cancel the nonautonomous part of (22), making the shape dynamics autonomous.

\section{CONVERGENCE ANALYSIS}

\section{A. Lyapunov function}

Consider the Lyapunov function candidate

$$
V_{\text {obst }}=-\ln \left(\mathbf{x}_{1} \cdot \mathbf{x}_{2}\right)+h(|\mathbf{r}|),
$$

where $h(\cdot)$ is continuously differentiable and satisfies certain hypotheses, to be specified below. In (23), the term $-\ln \left(\mathbf{x}_{1} \cdot \mathbf{x}_{2}\right)$ penalizes misalignment of the tangent vector of the moving vehicle with the tangent vector to the boundary curve at the closest point (and this term also blows up as $\mathbf{x}_{1} \cdot \mathbf{x}_{2} \rightarrow 0$, i.e., when (11) is violated). The term $h(|\mathbf{r}|)$ in (23) deals with the separation between the moving vehicle and the obstacle. We assume that

$$
\lim _{\rho \rightarrow 0} h(\rho)=\infty
$$

so that $V_{\text {obst }}$ blows up as the moving vehicle approaches collision with the boundary curve.

The time derivative of $V_{\text {obst }}$ along trajectories of (4) is

$$
\begin{aligned}
\dot{V}_{\text {obst }} & =-\frac{\left(\mathbf{x}_{1} \cdot \dot{\mathbf{x}}_{2}+\dot{\mathbf{x}}_{1} \cdot \mathbf{x}_{2}\right)}{\mathbf{x}_{1} \cdot \mathbf{x}_{2}}+f(|\mathbf{r}|) \frac{d}{d t}|\mathbf{r}| \\
& =-\frac{\left[\left(\mathbf{x}_{1} \cdot \mathbf{y}_{2}\right) u_{2}+\nu_{1} \kappa_{1}\left(\mathbf{x}_{2} \cdot \mathbf{y}_{1}\right)\right]}{\mathbf{x}_{1} \cdot \mathbf{x}_{2}} \mp f(|\mathbf{r}|)\left(\mathbf{x}_{1} \cdot \mathbf{y}_{2}\right) \\
& =-\frac{\mathbf{x}_{1} \cdot \mathbf{y}_{2}}{\mathbf{x}_{1} \cdot \mathbf{x}_{2}}\left[u_{2}-\nu_{1} \kappa_{1} \pm f(|\mathbf{r}|)\left(\mathbf{x}_{1} \cdot \mathbf{x}_{2}\right)\right]
\end{aligned}
$$

where $f(\rho)=d h / d \rho$, and we have used

$$
\mathbf{x}_{2} \cdot \mathbf{y}_{1}=-\mathbf{x}_{1} \cdot \mathbf{y}_{2} .
$$

One possible choice for $f(\cdot)$ is

$$
f(|\mathbf{r}|)=\alpha\left[1-\left(\frac{r_{o}}{|\mathbf{r}|}\right)^{2}\right],
$$

where $\alpha$ and $r_{o}$ are positive constants, and $r_{o}$ represents the desired separation between the moving vehicle and the boundary curve for boundary-following.

\section{B. Choice of control law}

One choice of $u_{2}$ which leads to $\dot{V}_{\text {obst }} \leq 0$ is

$$
\begin{aligned}
u_{2} & =\mu\left(\mathbf{x}_{1} \cdot \mathbf{y}_{2}\right) \mp f(|\mathbf{r}|)\left(\mathbf{x}_{1} \cdot \mathbf{x}_{2}\right)+\nu_{1} \kappa_{1} \\
& =\mu\left(\mathbf{x}_{1} \cdot \mathbf{y}_{2}\right)-f(|\mathbf{r}|)\left(\frac{\mathbf{r}}{|\mathbf{r}|} \cdot \mathbf{y}_{2}\right)+\nu_{1} \kappa_{1} \\
& =\mu\left(\mathbf{x}_{1} \cdot \mathbf{y}_{2}\right)-f(|\mathbf{r}|)\left(\frac{\mathbf{r}}{|\mathbf{r}|} \cdot \mathbf{y}_{2}\right)+\left(\frac{\mathbf{x}_{1} \cdot \mathbf{x}_{2}}{1 \pm\left|\kappa_{1}\right||\mathbf{r}|}\right) \kappa_{1} .
\end{aligned}
$$

where $\mu>0$ is a constant (or a function of $|\mathbf{r}|$ ), and where our convention for the positive direction of the boundary curve has also been incorporated into $\kappa_{1}$.

Expression (28) is simple to interpret. The term $\mu\left(\mathbf{x}_{1} \cdot \mathbf{y}_{2}\right)$ serves to align the moving vehicle with the tangent vector to the closest point on the boundary curve. The term involving $f(\cdot)$ serves to steer the moving vehicle towards or away from the boundary curve to achieve the desired separation. Finally, the term involving $\kappa_{1}$ enables the moving vehicle to respond to the nonzero curvature of the boundary curve.

This way of thinking about obstacle avoidance and boundary following leads to the idea that the role of the sensors on the moving vehicle is to identify the closest point on the boundary curve, and to estimate the curvature of the boundary at that point. Once we have identified (e.g., initially) the closest point, we can then, in principle, track the closest point as it and the moving vehicle trajectory evolve. However, we also need to periodically scan in other 
directions, in case the local minimum we are tracking ceases to be a global minimum at some point in time.

\section{Shape-variable convergence}

Observe that (28) is well-defined even for non-constant $\kappa_{1}$ (unless $|\mathbf{r}|=1 /\left|\kappa_{1}\right|$ and the boundary curves inward toward the moving vehicle). But to simplify the convergence analysis, we now take $\kappa_{1}=$ constant. The Lyapunov function expressed in the shape variables $(\rho, \phi)$ is

$$
V_{\text {obst }}=-\ln (\cos \phi)+h(\rho),
$$

and the steering control $u_{2}$ for the moving vehicle is

$$
\begin{aligned}
u_{2} & =\mu \sin \phi \mp f(\rho) \cos \phi+\nu_{1} \kappa_{1} \\
& =\mu \sin \phi \mp f(\rho) \cos \phi+\left(\frac{\kappa_{1}}{1 \pm\left|\kappa_{1}\right| \rho}\right) \cos \phi .
\end{aligned}
$$

The time derivative of $V_{\text {obst }}$ along trajectories of (19) and (22), with $u_{2}$ given by (30) is then

$$
\begin{aligned}
\dot{V}_{\text {obst }} & =-\frac{\sin \phi}{\cos \phi}\left[u_{2}-\nu_{1} \kappa_{1} \pm f(\rho) \cos \phi\right] \\
& =-\mu \frac{\sin ^{2} \phi}{\cos \phi} \\
& \leq 0,
\end{aligned}
$$

due to (18). Thus, $\dot{V}_{\text {obst }} \leq 0$ and $\dot{V}_{\text {obst }}=0$ if and only if $\sin \phi=0$. But by (18), we see that $\dot{V}_{\text {obst }}=0$ if and only if $\phi=0$. This calculation leads to the following result.

Proposition 1: Consider the dynamics (4) with control law (28) for a moving vehicle and the closest point to it on the boundary curve. Assume that the boundary curve is a circle (i.e., $\kappa_{1}=$ constant $\neq 0$ ), the moving vehicle initially lies outside this circle, and initially (11) is satisfied. Assume also that

(A1) $d h / d \rho=f(\rho)$, where $f(\rho)$ is a Lipschitz continuous function on $(0, \infty)$, so that $h(\rho)$ is continuously differentiable on $(0, \infty)$;

(A2) $f(\rho)=0$ at a finite number of isolated points;

(A3) $\lim _{\rho \rightarrow 0} h(\rho)=\infty, \lim _{\rho \rightarrow \infty} h(\rho)=\infty$, and $\exists \tilde{\rho}$ such that $h(\tilde{\rho})=0$.

(A4) $\mu(\rho)>0$ is a Lipschitz continuous function on $(0, \infty)$.

Then the system (4) with (28) converges to a limit cycle solution in which the moving vehicle follows a circular orbit with the same center as the circular boundary curve, and at a distance from the boundary curve given by one of the zeros of the function $f(\cdot)$.

Proof: Note that as long as the moving vehicle is outside of the circular boundary curve, the closest point is welldefined and is unique. (In fact, the closest point lies on the line connecting the center of the circular obstacle with the moving vehicle.) Furthermore, $u_{2}$ is well-defined, and in particular,

$$
\nu_{1}=\frac{\mathbf{x}_{1} \cdot \mathbf{x}_{2}}{1+\left|\kappa_{1}\right||\mathbf{r}|}=\frac{\cos \phi}{1+\left|\kappa_{1}\right| \rho}
$$

so that the denominator is bounded away from zero. Here $\phi$ is defined by (16) and $\rho=|\mathbf{r}|$. Since (11) holds initially, so does (18).

Observe that $V_{\text {obst }}$ given by equation (29) is continuously differentiable provided (18) holds. By assumption (A3) and the form of $V_{o b s t}$, we conclude that $V_{o b s t}$ is radially unbounded (i.e., $V_{\text {obst }} \rightarrow \infty$ as $|\phi| \rightarrow \pi / 2$, as $\rho \rightarrow 0$, or as $\rho \rightarrow \infty)$. Therefore, for each trajectory that initially satisfies (18) and $\rho>0$, there exists a compact sublevel set $\Omega$ of $V_{\text {obst }}$ such that the trajectory remains in $\Omega$ for all future time. Then by LaSalle's Invariance Principle [7], the trajectory converges to the largest invariant set $M$ of the set $E$ of all points in $\Omega$ where $\dot{V}_{\text {obst }}=0$. The set $E$ in this case is the set of all points in $\Omega$ such that $\sin \phi=0$; i.e., such that $\phi=0$. At points in $E$, the dynamics may be expressed as

$$
\begin{aligned}
& \dot{\rho}=0, \\
& \dot{\phi}= \pm f(\rho) .
\end{aligned}
$$

If $f(\rho) \neq 0$, then the trajectory leaves $E$. Therefore, the largest invariant set contained in $E$ may be expressed as

$$
M=\{(\rho, \phi) \mid \phi=0, \quad f(\rho)=0\} .
$$

By assumption (A2), $M$ consists of isolated equilibria, and therefore, we can conclude that $(\rho, \phi)$ converges to an equilibrium. This equilibrium in the $(\rho, \phi)$ variables amounts to the moving vehicle following a circular orbit with the same center as the boundary curve, and at a distance from the boundary curve given by one of the zeros of the function $f(\cdot)$.

Proposition 2: Consider the dynamics (4) with control law (28) for a moving vehicle and the closest point to it on the boundary curve. Assume that the boundary curve is a straight line (i.e., $\kappa_{1} \equiv 0$ ), initially the moving vehicle does not lie on the boundary curve, and initially (11) is satisfied. Also, assume (A1), (A2), (A3), and (A4) of Proposition 1 above are satisfied. Then the system converges to a relative equilibrium (i.e., an equilibrium of the shape dynamics) in which the moving vehicle follows a linear path parallel to the boundary curve, and at a distance from the boundary curve given by one of the zeros of the function $f(\cdot)$.

Proof: Identical to the proof of Proposition 1. We conclude that $(\rho, \phi)$ converges to an equilibrium. This equilibrium in the $(\rho, \phi)$ variables amounts to the moving vehicle following a straight-line path parallel to the boundary curve, and at a distance from the boundary curve given by one of the zeros of the function $f(\cdot)$.

\section{BOUNDARY FOLLOWING IN THREE DIMENSIONS}

One of the major strengths of our planar boundaryfollowing law is that it can be generalized to the threedimensional setting. Suppose there is a vehicle moving in three-dimensional space, and there is a fixed, smooth, twodimensional obstacle surface (e.g., a sphere). As the moving 
vehicle moves at unit speed, the closest point (which we assume is unique) also moves along a three-dimensional trajectory, but is constrained to lie on the obstacle surface. We can then, as in the planar problem, consider the coupled dynamics of the moving vehicle and the closest point.

Instead of (4), we have

$$
\begin{array}{ll}
\dot{\mathbf{r}}_{1}=\nu_{1} \mathbf{x}_{1}, & \dot{\mathbf{r}}_{2}=\mathbf{x}_{2}, \\
\dot{\mathbf{x}}_{1}=\mathbf{y}_{1} u_{1}+\mathbf{z}_{1} v_{1}, & \dot{\mathbf{x}}_{2}=\mathbf{y}_{2} u_{2}+\mathbf{z}_{2} v_{2}, \\
\dot{\mathbf{y}}_{1}=-\mathbf{x}_{1} u_{1}, & \dot{\mathbf{y}}_{2}=-\mathbf{x}_{2} u_{2}, \\
\dot{\mathbf{z}}_{1}=-\mathbf{x}_{1} v_{1}, & \dot{\mathbf{z}}_{2}=-\mathbf{x}_{2} v_{2},
\end{array}
$$

where:

- $\mathbf{r}_{1}$ is the position (in $\mathbb{R}^{3}$ ) of the closest point;

- $\mathbf{x}_{1}$ is the unit tangent vector to the trajectory of the closest point

- $\mathbf{y}_{1}$ and $\mathbf{z}_{1}$ are unit normal vectors which complete (with $\mathbf{x}_{1}$ ) a right-handed orthonormal frame;

- $\left(u_{1} / \nu_{1}, v_{1} / \nu_{1}\right)$ are the natural curvatures associated to the normal development of the trajectory followed by the closest point [8];

- $\mathbf{r}_{2}$ is the position (in $\mathbb{R}^{3}$ ) of the moving vehicle;

- $\mathbf{x}_{2}$ is the unit tangent vector to the trajectory of the moving vehicle;

- $\mathbf{y}_{2}$ and $\mathbf{z}_{2}$ are unit normal vectors which complete (with $\mathbf{x}_{2}$ ) a right-handed orthonormal frame; and

- $u_{2}$ and $v_{2}$ are the controls which determine the trajectory followed by the moving vehicle.

Here we are interested in determining the controls $u_{2}$ and $v_{2}$ for the moving vehicle as feedback functions of the other variables, for the purpose of achieving non-collision and boundary-following.

Figure 2 illustrates the frames for the trajectories of the moving vehicle and the closest point on the boundary surface.

In (35), we have used the natural Frenet frame (or Fermi-Walker frame or relatively-parallel adapted frame) representation for describing a (twice continuously differentiable) curve [8], [9]. This description is unique up to an arbitrary initial choice of the unit-normal-vector orientation in the normal plane. The Frenet-Serret frame, which is more widely mentioned than the natural Frenet frame, exists for a three-times continuously differentiable curve with non-vanishing curvature, and is unique. In the boundaryfollowing analysis (as well as for interaction laws of unitspeed vehicles [6]), it seems to be advantageous to use the natural Frenet frame, rather than the Frenet-Serret frame.

As in the planar problem, we define $\mathbf{r}=\mathbf{r}_{2}-\mathbf{r}_{1}$ to be the relative position of the moving vehicle with respect to the closest point, and we assume that initially $|\mathbf{r}|>0$. Equations (7) and (8) hold, as in the planar case, however (9) need not. As in the planar case, we assume that initially the moving vehicle is not heading directly toward or directly away from the closest point, so $\mathbf{x}_{1}$ may be chosen such that (11) holds.

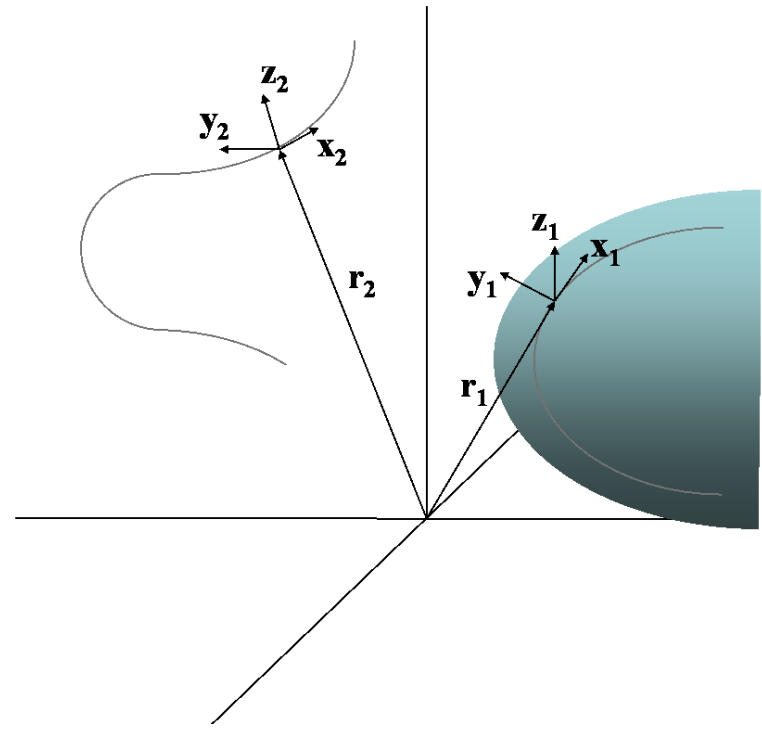

Fig. 2. Positions and frames for the trajectory of the moving vehicle $\left(\mathbf{r}_{2}\right.$, $\left.\mathbf{x}_{2}, \mathbf{y}_{2}, \mathbf{z}_{2}\right)$ and for the closest point on the boundary surface $\left(\mathbf{r}_{1}, \mathbf{x}_{1}\right.$, $\left.\mathbf{y}_{1}, \mathbf{z}_{1}\right)$.

Although the two-dimensional obstacle surface is assumed to be specified (or sensed locally at the closest point, e.g., using a laser rangefinder), further calculation (or sensing) is required to determine the trajectory of the closest point, and in particular, the natural curvatures $\left(u_{1} / \nu_{1}, v_{1} / \nu_{1}\right)$. The natural curvatures (which are functions of arc-length parameter) [8], determine how the trajectory of the closest point evolves, and in this problem, the natural curvatures for the closest-point trajectory are in turn determined by the shape of the obstacle surface and the motion of the moving vehicle. The natural curvatures for the closestpoint trajectory can be estimated from measurements of several points on the trajectory (although the details are beyond the scope of this paper).

A convergence result (which will appear in a future paper) can then be proved, using the same Lyapunov function candidate (23) as before, for a three-dimensional boundaryfollowing law and a spherical obstacle.

\section{Simulation RESUlTS}

Figures 3 and 4 show a moving vehicle tracking an elliptical boundary curve in the planar setting. In figure 3, the robot starts at coordinates $(-11,0)$ with initial orientation +10 degrees measured counterclockwise from the $\mathrm{x}$-axis. The closest point then lies to the right of the robot. Under our control law, the robot follows the boundary curve to its right, circling the obtacle clockwise. In figure 4, the robot starts at the same initial position, but with the initial orientation -10 degrees. Then the robot follows the boundary curve to its left, circling the obstacle counterclockwise. In both cases, the desired separation between the robot and the closest points are set to 1 distance unit. 


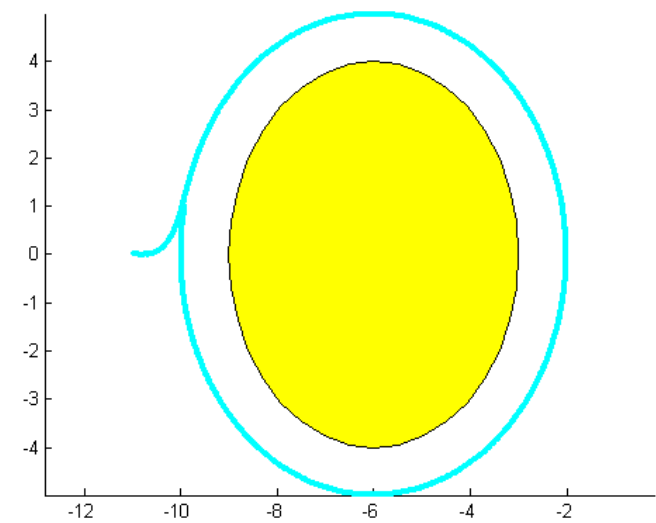

Fig. 3. Simulation results showing clockwise circling of an elliptical obstacle boundary using (28).

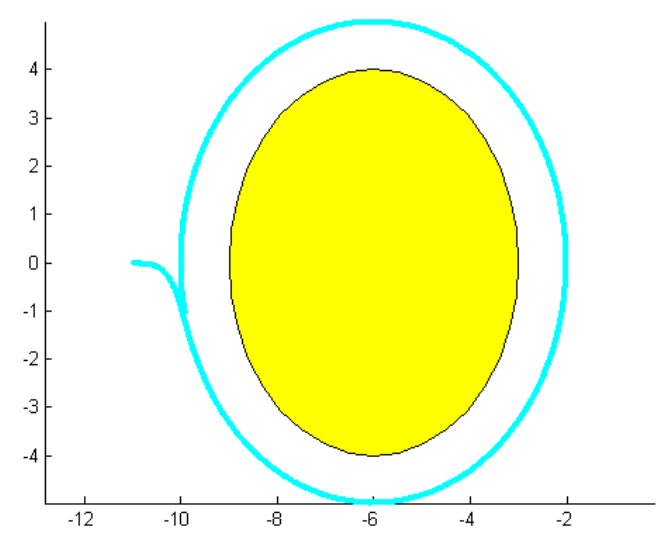

Fig. 4. Simulation results showing counterclockwise circling of an elliptical obstacle boundary using (28).

\section{DIRECTIONS FOR FUTURE RESEARCH}

The gyroscopic feedback laws for boundary following presented in this paper have several highly attractive features. First, only the heading of the vehicle is controlled, with the speed held constant, so that the laws are appropriate for vehicles which must maintain high speeds (including, e.g., unmanned aerial vehicles). Second, although at first glance the control laws may appear complicated, in fact, they mainly involve dot products of various unit vectors (i.e., sines and cosines of various angles), functions of distance between the vehicle and the closest point, and functions of the boundary curvature. Indeed, we have sought the simplest gyroscopic feedback laws that produce the desired boundary-following behavior. Third, the way in which sensor data is used is straightforward and simple to characterize. The sensors track the closest point on the boundary, and measure the curvature there. (Of course, as mentioned earlier, some wide-angle scanning would also be required for the practically important situation of nonunique closest points.)

There are various directions for future research, including: incorporating obstacle avoidance and boundary following with navigation toward a waypoint, incorporating influences from multiple obstacles into the control for a single moving vehicle, and incorporating both multiple obstacles and waypoint navigation into formation control laws for two or more moving vehicles. Understanding the most basic problems well, such as the interaction of a single vehicle with a single obstacle, represents a first step toward dealing with these more complicated situations. Accordingly, we plan to further develop the three-dimensional boundaryfollowing analysis, as well as to consider more general obstacle geometries in the planar setting. Here concepts such as Bertrand curves, (or relatively parallel curves) [8], [10] may play a role in characterizing the steady-state trajectories of the moving vehicle and closest point for general obstacle shapes.

\section{ACKNOWLEDGEMENTS}

This research was supported in part by the Naval Research Laboratory under Grants No. N00173-02-1G002, N00173-03-1G001, N00173-03-1G019, and N00173-041G014, by the Air Force Office of Scientific Research under AFOSR Grants No. F49620-01-0415 and FA95500410130, and by the Army Research Office under ODDR\&E MURI01 Program Grant No. DAAD19-01-1-0465 to the Center for Communicating Networked Control Systems (through Boston University). P. S. Krishnaprasad also gratefully acknowledges the support of the Bernoulli Center at EPFL, Lausanne.

\section{REFERENCES}

[1] L. Singh, H. Stephanou, and J. Wen, "Real-time robot motion control with circulatory fields," Proc. IEEE Int. Conf. Robotics and Automation, Vol. 3, pp. 2737-2742, 1996.

[2] F. Zhang, A. O'Connor, D. Luebke, and P.S. Krishnaprasad, "Experimental study of curvature-based control laws for obstacle avoidance," Proc. IEEE Int. Conf. Robotics and Automation, Vol. 4, pp. 38493854, 2004.

[3] D.E. Chang, S. Shadden, J.E. Marsden, and R. Olfati-Saber, "Collision avoidance for multiple agent systems," Proc. IEEE Conf. Decision and Control, Vol. 1, pp. 539-543, 2003.

[4] B.R. Fajen and W.H. Warren, "Behavioral dynamics of steering, obstacle avoidance, and route selection," Journal of Experimental Psychology: Human Perception and Performance, Vol. 29, No. 2, pp. 343-362, 2003.

[5] E.W. Justh and P.S. Krishnaprasad, "Equilibria and steering laws for planar formations," Systems and Control Letters, Vol. 52, pp. 25-38, 2004.

[6] E.W. Justh and P.S. Krishnaprasad, "Formation control in three dimensions," preprint, 2004.

[7] H. Khalil. Nonlinear Systems. New York: Macmillan Publishing Co., 1992.

[8] R.L. Bishop, "There is more than one way to frame a curve," The American Mathematical Monthly, Vol. 82, No. 3, pp. 246-251, 1975.

[9] A. Calini, "Recent developments in integrable curve dynamics," In Geometric Approaches to Differential Equations, Lecture Notes of the Australian Math. Soc., Vol. 15, p. 56-99, Cambridge University Press, 2000.

[10] R.S. Millman and G.D. Parker, Elements of Differential Geometry, Englewood Cliffs, NJ: Prentice Hall, 1977. 